\title{
A comparison between two different conditions of breaking up sedentary behaviour on glucose metabolism
}

Edvard H. Sagelv ${ }^{1,2}$, Ulf Ekelund ${ }^{1}$, Trine Stensrud ${ }^{1}$

${ }^{1}$ Department of Sports Medicine, Norwegian School of Sport Sciences, Norway. ${ }^{2}$ School of Sport Sciences, UiT the Arctic University of Norway, Norway. (Sponsor: Jorunn Sundgot-Borgen, FACSM)

\section{CONCLUSION}

There is no effect of either breaking up sedentary behaviour each hour or performing 30 minutes of vigorous physical activity in one bout compared with prolonged sitting for six hours on glucose metabolism.

\section{/ ABSTRACT}

INTRODUCTION: Sedentary behaviour is suggested a risk factor for various health outcomes, independent of the amount of subcomponents of physical activity. There is currently paucity of experimental studies comparing frequent breaks of sedentary time with one single long bout of exercise in an iso-caloric design. PURPOSE: to examine if breaking up sedentary behaviour with short five minutes' bouts or one continuous 30-minute bout of vigorous intensity activity differentially affects metabolic risk markers compared with a control condition of prolonged sitting.

METHODS: 12 healthy adults participated in a randomized cross-over design as follows;1) six hours of quiet sitting; 2 ) six hours of sitting, including five minutes exercise bouts (treadmill running) at $70 \%$ of maximal oxygen uptake $\left(\mathrm{VO}_{2 \max }\right.$ ) each hour; 3 ) continuous exercise at $70 \%$ of $\mathrm{VO}_{2 \max }$, for 30 minutes followed by five hours and 30 minutes of sitting. Prior to each test, a fasting blood sample was collected and followed by a standardized meal consisting of 646 kilocalories. Blood samples were thereafter collected every hour. Maximal aerobic power was measured using indirect calorimetry and the running speed equivalent to $70 \%$ of $\mathrm{VO}_{2 \max }$ was determined by extrapolation following a submaximal steady state exercise test prior to the experiment. Each condition was separated by a 6-day wash-out period. RESULTS: There were no differences in insulin (mean (95\% Cl); 1) 60704.8 (36352.4-79412.1) pmol • $\mathrm{L}^{-1} \bullet 360 \mathrm{~min}$; 2) 53375.7 (28787.871847.5) pmol • $\mathrm{L}^{-1} \bullet 360 \mathrm{~min}$; 3) 44827.3 (24853-67912.7) pmol $\bullet \mathrm{L}^{-1} \bullet$ $360 \mathrm{~min})$ and glucose (mean $(95 \% \mathrm{Cl})$; 1) $105(27.6-150) \mathrm{mmol} \bullet \mathrm{L}^{-1} \bullet$ $360 \mathrm{~min}$; 2) $48.4(-88.6-113.5) \mathrm{mmol} \bullet \mathrm{L}^{-1} \bullet 360 \mathrm{~min}$; 3) 105.8 (49.3171.6) $\mathrm{mmol} \bullet \mathrm{L}^{-1} \bullet 360 \mathrm{~min}$ ) incremental area under curve between conditions.

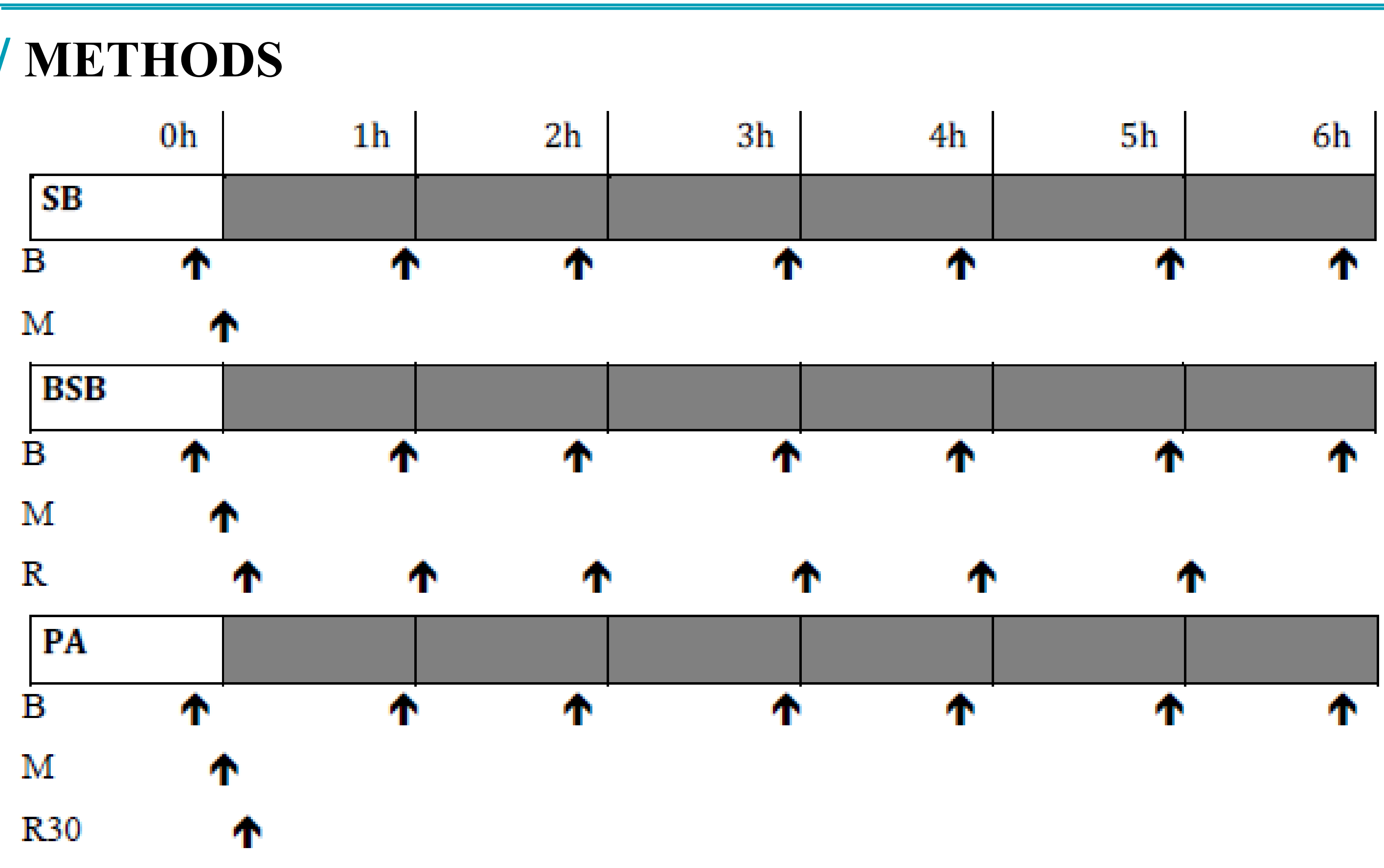

Figure 1: showing the protocol. $S B=S e$ dentary behaviour condition $B S B=$ Breaking sedentary behaviour condition, $P A=$ Physical Activity condition, $B=$ Blood samples, $M=$ Meal, $R=$ Run 5 minutes, $R 30=$ Run 30 minutes continiuously

\section{RESULTS}

Insulin

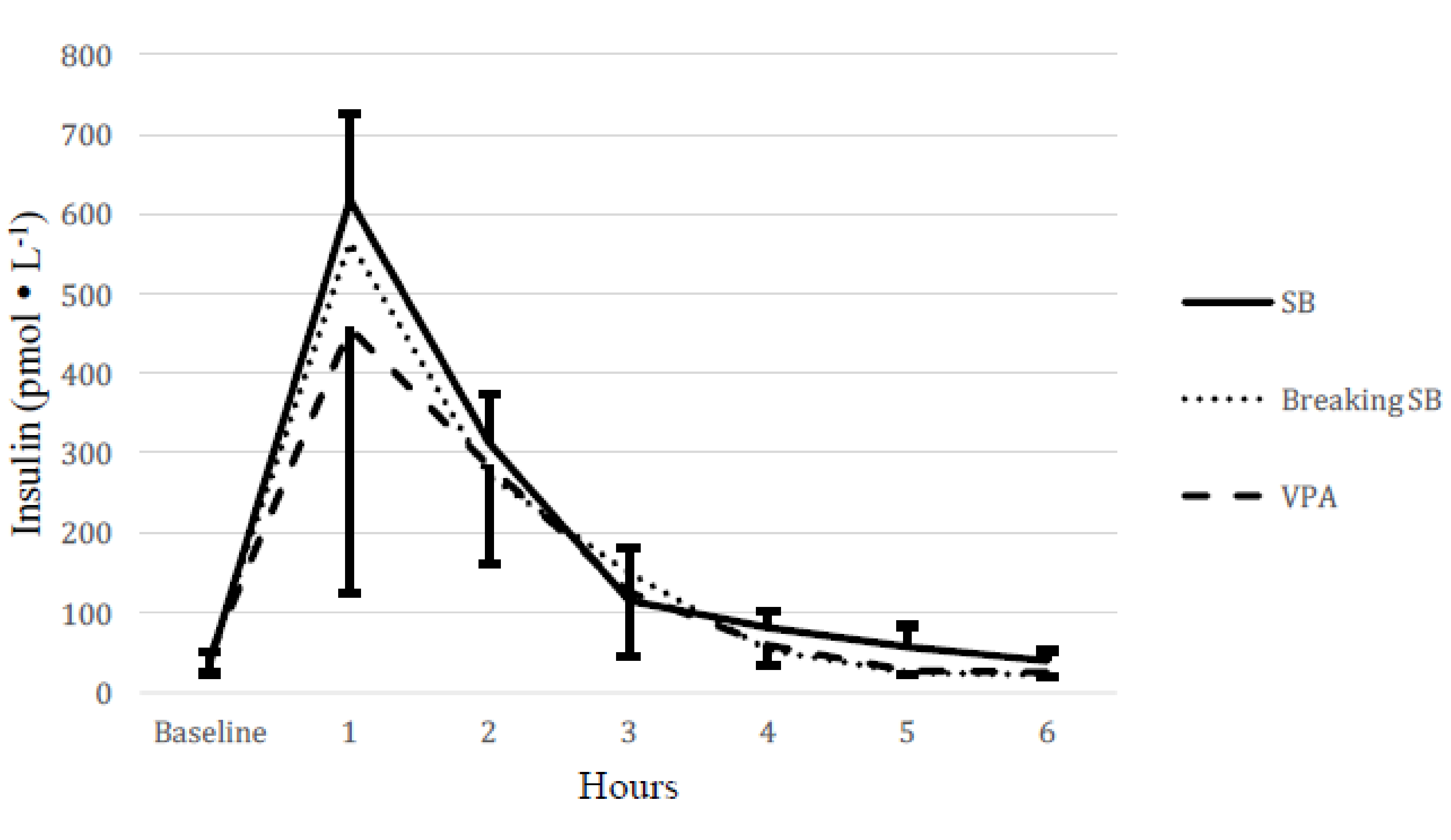

Figure 3: showing mean insulin concentration during six hours. Values are mean and error bars are $S D . S B=$ sedentary behaviour condition, Breaking $S B=$ Breaking sedentary behaviour condition, VPA = continuous vigorous physical activity condition.

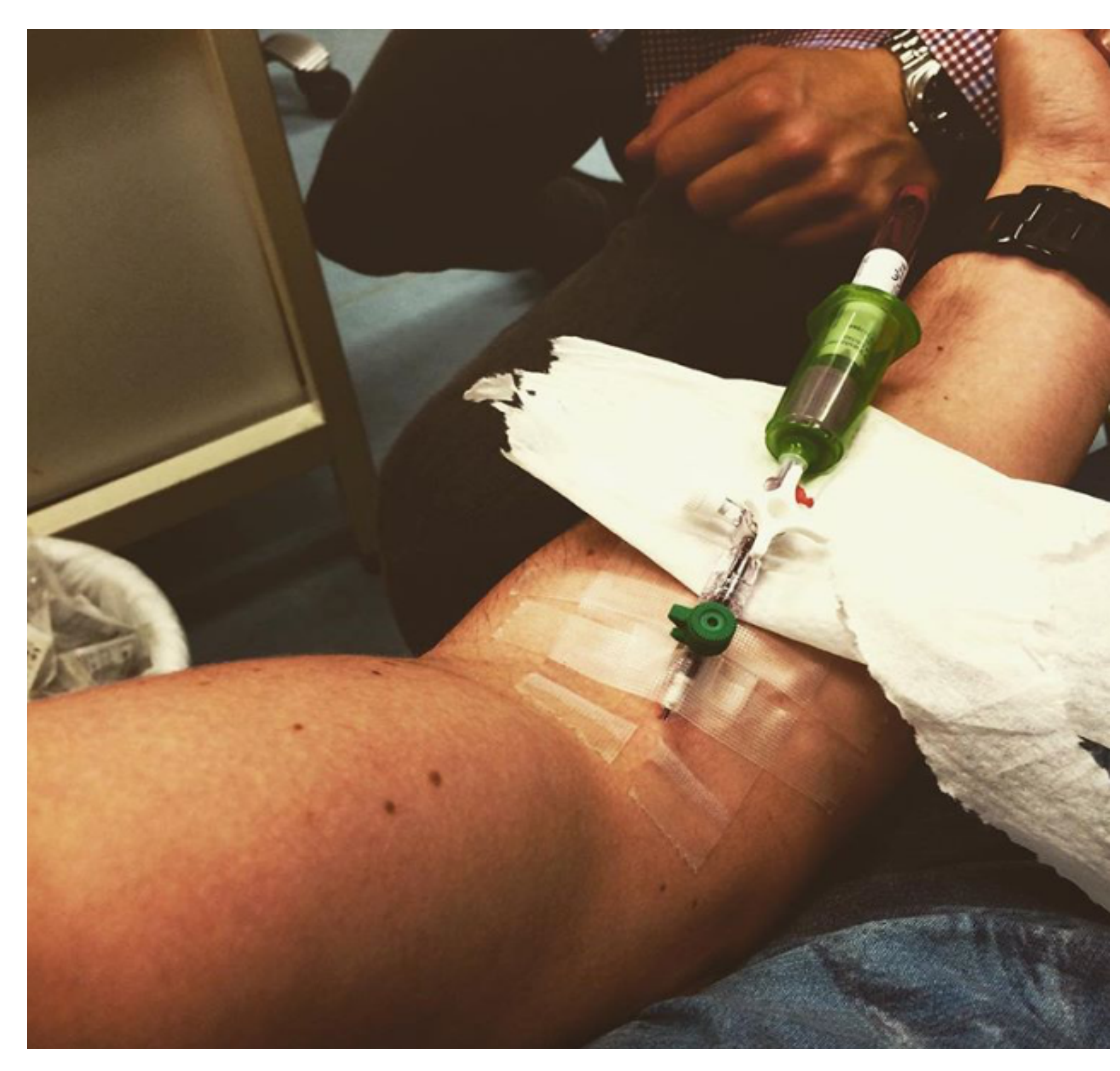

Figure 2: catheter inserted in the median cubital vein

conditions. 